\title{
13 Bubbles of Time
}

As we saw in Chapter 10, one of the consequences of Bell's theorem, confirmed by experimental evidence (in experiments carried out by Alain Aspect, Nicolas Gisin, and many others), is that time across the universe distributes itself in distinct spatial 'zones' within which time ticks to the same clock - time bubbles with shapes vaguely reminiscent, perhaps, of the ever-changing and ever-moving oil blobs of a 70's style lava lamp. The objects or entities inhabiting these zones make up the separatevariables of a valid collective wave function - which is how such bubbles of synchronous time create themselves in the first place (75). Whenever some change affects any one of the constituent variables, all the other variables react instantly so that the collective wave function seamlessly keeps being valid, irrespective of any spatial separation between the variables.

Now let's look at one of the consequences of the relativity of simultaneity. In the inimitable words of Paul Davies:

\begin{abstract}
If reality is vested in the present, then you have the power to change this reality across the universe, back and forth in time, simply by walking around. But then, so does an Andromedan sentient green blob. If the blob oozes to the left and then to the right, the present moment on Earth, as judged by the blob, in its frame of reference, will lurch through huge changes back and forth in time.
\end{abstract}

There is only one rational conclusion from the relative nature of simultaneity: events in the past and future are every bit as real as events in the 'present': the distinction between past, present and future is bereft of meaning (76).

By tweaking distances and speeds, we can grow at will such separation to decades (77). So far, so good: nothing much new here.

But the description above of oozing to the left or to the right, or earlier of 'getting up and walking' is just too macroscopic and vague.

To appreciate this, let's act out the experiment that was touched upon in the Prologue: point out the forefinger of your right hand and move your right arm in the direction of the Andromeda galaxy in the constellation Cassiopeia, while you stretch out your left forefinger and simultaneously move your left forefinger towards any galaxy located in the diametrically opposed corner of the sky - say, the constellation Crux (aka the Southern Cross.)

Quite oddly, different parts of your body have now become separately simultaneous with different events, unfolding far away. These events are days apart from one another.

Let's make it a bit more puzzling. Start spinning on your heels, around your body's up/down axis. As you spin, one side of your brain moves closer to Andromeda while at the same time the other side of your brain moves towards the Southern Cross: you now appear to have become a split individuality in terms of which time bubble you inhabit. 
But you are not: you happen to be whole, hale and sound. Your left brain is simultaneous with your right brain. At the very least, your two brain halves are not living days apart from each other. In a word, you are unitary. Yet as you spin, your left brain is simultaneous with events that are days apart from other events your right brain is simultaneous with.

There are only a few ways this apparent contradiction can be resolved: maybe your whole persona - who you are - is not distributed throughout the brain: your whole persona is contained in a space no bigger than the smallest quantum time cells we discovered earlier. Every time cell within your brain stays within its own determinate time. It never spreads out and simultaneity is always uniquely definable. But this seems absurd: the brain itself, or alternatively the minimum volume of space it needs to enable and perform the information processing that takes place within it, is vastly bigger than an elementary time cell.

As we saw earlier, Bell entanglement may exist within the brain: the various constituents and functional parts of the brain are instantly correlated (as are quite possibly all the other parts of your body, such as the fingertips of your spatially separate two hands): essentially because they are all participant elements in a single wave function, following the dictates of a valid Schrödinger equation, they operate in sync under a same time clock. When we spin, i.e. when we move our brain or body parts at different speeds towards different directions, it does not matter from the standpoint of what our governing time is: our brain's functional constituents all operate within the same clock time zone. A healthy brain has its own wave function, and all of its constituent parts are fully entangled within that wave function. A brain, as per Bell's theorem, inhabits its own same-time bubble.

But walking towards Andromeda (or spinning, thus alternatively nearing and receding from it) leads to a here-and-now simultaneity with that galaxy that ceaselessly shifts back and forth by about two whole days. Similarly, it's only a matter of calculation by what amount our simultaneity changes with respect to much closer places, such as for instance the next room in your house.

Indeed, if it were not for the instantaneous time enabled by Bell's theorem, the only possible simultaneity with our here-and-now would occur with places close enough such that the simultaneity shift would last less than a reigning quantum of time. If that quantum of time were, say, the Planck time we encountered earlier, then the distance corresponding to unique time compatible with any motion at usual walking or spinning speeds would be much less than the size of an atom (78). Therefore, the only way we can ever stay synchronous with any thing at all out there is if we are a participant in a collective wave function containing that thing as a participatory variable.

Many physicists conclude from the above that fundamentally, time cannot exist (see also 75). But is there an elephant in the room? 
Let's recap where we are:

First, let's pick a person whose brain is self-coherent, and therefore operates wholly within its own simultaneous time, in other words within a 'Bell' time bubble. This person's wave function is coherent.

Second, because of relativistic effects, this person (79) can apparently have different areas of her otherwise unique-time-clock brain being simultaneously simultaneous, or simultaneous at the same time, with events and entities which are however separated between themselves by days.

It is the combination of the two points above that has led many Physicists to conclude, as we have seen, that the distinction between past, present, and future is bereft of meaning: you cannot be simultaneous at the same time with two events separated in time. Something has to yield. For a number of physicists, it is time itself that yields.

But this might not be the full explanation.

The elephant in the room is the way how and why things become entangled to begin with, i.e. become associated within a coherent common wave function.

Think of some piece of wood on Earth which may happen to be moving, buffeted by the wind, but which no one sees, no one perceives, no one cares about or knows about in any way. To that piece of wood, anything or anyone anywhere in Andromeda simply does not exist, does not come near any relationship or cognition or linkage of any conceivable kind. We cannot in any meaningful sense speak of correlation or simultaneity of this piece of wood with anything in Andromeda. There is zero correlation.

Unless some consciousness looks at that piece of wood and establishes a mental picture of its simultaneity with something there. Somewhere inside a mind, a link has been forged.

Likewise, in the previous picture, the person was sitting and thinking of Andromeda, or of something somewhere in Andromeda. That established a 'rapport' of some kind, where none existed before. In the case of the wood, as in the case of the person, as in the case of the two correlated particles in Alain Aspect's and Nicolas Gisin's and all other experiments that confirmed Bell's theorem, a consciousness was present to establish some correlation, some linkage between the participatory elements - elements which otherwise would have been fully ignorant of each other (or of one another) - entities passing each others unawares like distant ships in the night, in disjointed and distinct Bell time bubbles.

A consciousness poured water into a teapot and thereby created a wave function associated with the full teapot, and then it interfered with that newly-minted wave function by watching it.

Consciousness could be seen as having created, either indirectly but instrumentally in the case of Alain Aspect's et al. labs, or directly, in the case of the seated thinking person, a new wave function; building on and coalescing hitherto separate individual wave functions. This new wave function evolved from existing 
wave functions, such as the person's mind, and expanded to encompass more variables. Doing so, it partly cohered towards another wave function, such as that of some object on Andromed; it combined the thinking person on their chair on Earth, with the entity in Andromeda that they were thinking of. That person can now spin, move their fingers at speed towards opposite directions - a Bell time bubble binding them and the object of their thoughts in Andromeda has formed, and simultaneity has now set in. The person can spin and move different parts of their body and brain towards different azimuths and stay whole: the contradiction has now vanished.

But there is no qualitative difference whatsoever between a wave function relating to several variables, or one relating to only one variable. These wave functions are of the exact same essence. Indeed, underscoring their common nature, any wave function of a multi-variable, or multi-constituent system, can always be expressed by a mathematical expression that includes a product (in essence, a multiplication involving 'imaginary' numbers) of the erstwhile individual wave functions of its constituents (80) : whatever gives rise to a multi-component wave function must also give rise to a single-variable one.

Specifically, if a multi-variable wave function demands the intervention of a volition to exist, then so does a one-variable wave function: whatever gives rise to the former must also give rise to the latter. It follows that if the existence of multicomponent wave functions requires the intervention of a consciousness, then individual, one-constituent wave functions must also be created by a consciousness.

Therefore the Big Bang itself, which created a materiality steered and governed by wave functions, could not have happened without the existence of some prior consciousness - i.e., some sentient being (81).

We could call this consciousness Tom, (for 'the original mind') or alternatively, because we do not know if there is only one Tom, OM for 'Original Mind(s)', and we'll analyze it further in Chapter 15.

Before we can do that, let's examine some possible objections.

A rather odd objection that was put forward was that the above quote proves the existence of God unquote. Apart from the odd and rather unscientific thought that if something actually proves something contrary to one's views, then it is an argument against the proof, it actually does nothing of the kind: the requisite pre-existing consciousness could be any consciousness in a pre-existing matrix universe whence our universe was born: say, a software engineer (in the 'stimulated reality scenarios' which we'll revisit shortly), or a lab assistant, or such (including in the case where our universe would have been born from a black hole; in the universe genesis by black holes scenario, not only our universe could have been created by a black hole from within an earlier universe, but our universe itself might also be continually spawning new universes. In that scenario, our consciousness would be enough to help create new universes), or any other consciousness. We will revisit these issues when we look at various multiverse scenarios (82). Beyond that, possible scenarios involve the possibility of the universe bootstrapping itself, owing to quirks in the topology of spacetime, as we shall see shortly. 
The more interesting objections all boil down to the same objection: the use of math to underpin the whole argument. This constitutes, again, the inescapable kernel of the matter. At the very least, it seems that we can more readily comprehend time and the universe itself if it is a mathematical structure. There seem to be no ways however complex to understand the universe if the contrary Aristotelian view is right, if matter is the ultimate reality and all our math nothing but glorified bean-counting tools.

It's time we looked at the various multiverse scenarios. 